\title{
Sensor Networks over Information Fields: Optimal Energy and Node Distributions
}

\author{
Hithesh Nama and Narayan Mandayam \\ WINLAB, Rutgers University \\ Piscataway, NJ 08854 \\ Email: \{hithesh, narayan\}@winlab.rutgers.edu
}

\begin{abstract}
Wireless sensor networks are typically deployed over an information field to sense and gather information from a distributed physical process. Resource allocation problems considered in the literature often ignore the underlying information field and rather consider a uniform distribution of information. In this paper, we propose an information field model that partitions the observation space into a grid, with independent information being generated at each point in the grid. Given this model, we find the optimal node distribution over the field that maximizes the network information capacity or the total information gathered over the lifetime of the network. The optimal node distribution is obtained by considering the equivalent problem of optimal energy distribution and flow over the information field that maximizes the information capacity.
\end{abstract}

\section{INTRODUCTION}

With advances in miniaturization of radio transceivers and their integration with low-power computing platforms, large scale networks of tiny wireless sensors are becoming a reality. It is envisaged that these networks will be useful in environmental monitoring, factory automation, in hostile environments like battlefields, and in disaster management. While the size of these sensors and their diminishing cost enable deployment in large numbers, the flip side is that these sensors are highly energy constrained and battery replacement is prohibitive in most applications. Thus the biggest challenge in building sensor networks is the development of power-aware protocols - for discovery, routing, scheduling, and physical layer communication - that maximize the operational lifetime of these networks.

Typically, sensor networks are deployed over a particular geographic region or an information field and the task of the sensors is to extract information about a distributed physical process in the field, and collaboratively forward it to a set of collector or sink nodes. In the rest of the paper we use the term information field to also refer to the physical process being sensed. It is important to know the characteristics of the underlying information field in order to evaluate the performance of a sensor network in terms of its information capacity, which is the total information gathered over the lifetime of the network. In this paper, we introduce the notion of an information-rate density function based on the entropy rate of the underlying physical process. This density function is used to characterize the information field over which the

This work is funded in part by the NJ Commission on Science and Technology under the MUSE project. sensor network is deployed. The data rates of the sensor nodes are governed by the information-rate density and the density of nodes in the field.

Much of the early work has focused on developing poweraware routing protocols ([1], [2], [3]) and topology control algorithms ([4], [5]). The general approach followed was that of minimizing the total energy consumed in the network by forwarding packets over minimum energy paths. These paths were computed heuristically using distributed shortest path algorithms with edge costs representing the required transmission power. However such an approach can burden some of the nodes common to several shortest paths thus depleting them of their energy faster. In order to avoid such a problem, [1] and [3] also proposed routing algorithms with node costs factoring in the node energy reserves.

Rather than trying to minimize the energy consumed per packet using different edge and node costs, [6] and [7] directly address the problem of achieving the desired global objective, that of maximizing the network lifetime. The problem is formulated using a flow-based approach and is transformed into a linear program when network lifetime is defined as the time until the death of the first node. Reference [8] evaluates the performance of distributed shortest path algorithms with different heuristic costs, by quantifying their performance relative to the optimal linear program solution. In [9] the flowbased linear program formulation is extended to the case of aggregating networks.

In all of the literature mentioned above, the sensor nodes are assumed to have arbitrary data rates irrespective of the information field in which the network is deployed. In [10], the extreme case of a continuous deployment of sensors is considered and information-rate density is defined as the total information rate of the sensors per unit area. The authors also study the problem of finding an optimal node distribution in the field that maximizes the network lifetime. However, the optimization is performed only over a particular class of node distributions and is not general in nature. Reference [11] studies the maximum lifetime sensor deployment problem with coverage constraints. Most recently the optimal energy distribution has been studied in [12] in the context of limited energy networks.

In this paper, we study the problem of finding the optimal node distribution given the underlying information field model. We generalize the linear program formulation in [6] 
to find out both the optimal initial energy distribution over the information field and the optimal flow that together maximize the network lifetime. As discussed in Section IV, the optimal initial energy distribution provides a simple solution to the problem of finding the optimal node distribution in the information field, without having to consider different classes of node distributions as in [10].

In the next section we describe the system model used in the paper and in Section III we setup the problem of maximizing the lifetime given a total energy constraint among the nodes. Section IV contains a discussion and simulation results that illustrate the usefulness of the optimal energy distribution. We conclude in Section V.

\section{SySTEM MODEL}

When sensor nodes are deployed in an information field, each node samples the process in its vicinity and for a given distortion its data rate will be equal to the entropy rate of the random process measured at that location. Since information can be spatially correlated, the total information rate of the sensor network is equal to the joint entropy rate of the random processes measured at all the nodes. When a large number of sensor nodes are deployed such that every point in the field is within the sensing range of at least one sensor, then the joint entropy of all the nodes can be considered to be the total information rate of the field. Addition of any further nodes does not increase the total information rate of the network. We can now define an information-rate density function for the information field such that the net volume under the function is equal to the total information rate of the field. The informationrate density function is thus a fundamental characteristic of an information field. Specifically in this paper, we consider an information field model that partitions the observation space into a grid with independent information being generated at each point in the grid.

In our system model, we consider a network of static wireless sensors that gather data from an information field and collectively forward the data to a set of collector nodes within the network. In-network aggregation or compression of data from different sensors is not considered in this model. Thus each sensor node in the network acts as a source of data and as a relay for forwarding data from other sensors to the sink. The aggregate rate at which information is delivered to the collector nodes is thus equal to the sum of the source rates of all the sensors.

As in [6] we consider the pure routing problem and ignore the effects of interference among the nodes by assuming a wideband communication model. The lifetime of the network thus obtained is only an operational lifetime and will always be an upper bound on the lifetime of a network with interference among nodes factored in as well. At the physical layer, the channel is assumed to be an AWGN channel and link gains are proportional to $d^{-\alpha}$, where $d$ is the separation between the transmitter and the receiver and $\alpha$ is the path loss exponent. The nodes are assumed to transmit at the least power that would guarantee a fixed SNR at the receiver and thus a fixed bit error rate for a given modulation scheme. The receiver is a conventional matched filter based receiver.

The node energy dissipation model is similar to that proposed in [9]. Each node dissipates energy in sensing, transmitting, and receiving data. Energy dissipated in idle receiving is ignored. We assume that the link capacity requirements on each node are feasible in that each node is capable (if necessary) of carrying the burden of routing the combined information from all the other nodes in the network.

\section{PROBLEM Formulation}

Consider a directed graph $G(M, A)$ where $M$ is the set consisting of all nodes and $A$ is the set of directed links in the network. Let $C \subset M$ denote the subset of collector or sink nodes and $M-C$ the complementary subset of sensor nodes. The set of link flows is denoted by $\mathbf{f}=\left\{f_{i, j}\right\}$ where, $f_{i, j}$ is the rate of information transfer over the directed link $(i, j)$. Let $r_{i}$ be the rate at which information is generated at node $i$. The flow conservation constraint at each sensor node is given by

$$
\sum_{j \in M} f_{j, i}+r_{i}=\sum_{j \in M} f_{i, j}, \forall i \in M-C
$$

Let $\mathcal{E}_{t x}^{i, j}$ denote the cost per data-unit for transmitting over the link $(i, j), \mathcal{E}_{r x}$ be the cost per data-unit for receiving over any link, and $\mathcal{E}_{s}$ the cost per data-unit for sensing at any node. Let $\mathbf{E}=\left\{E_{i}\right\}$ be the set of initial energies of the sensor nodes with $E_{i}$ denoting the initial energy of node $i$. We assume a total initial energy constraint $\left(E_{\text {total }}\right)$ on the sensor nodes such that

$$
\sum_{\forall i \in M-C} E_{i} \leq E_{\text {total }}
$$

The lifetime of node $i$ under flow $\mathbf{f}$ and initial energy distribution $\mathbf{E}$ is,

$$
T_{i}(\mathbf{f}, \mathbf{E})=\frac{E_{i}}{\sum_{j \in M}\left\{f_{i, j} \cdot \mathcal{E}_{t x}^{i, j}+f_{j, i} \cdot \mathcal{E}_{r x}\right\}+r_{i} \cdot \mathcal{E}_{s}}
$$

and we define the lifetime of the network as,

$$
T_{n w k}(\mathbf{f}, \mathbf{E})=\min _{\forall i \in M-C} T_{i}(\mathbf{f}, \mathbf{E})
$$

i.e., the time until the death of the first sensor node. The maximum lifetime of the network over the set of all feasible flows and the set of all feasible initial energy distributions can be obtained as

$$
\max _{\mathbf{f}, \mathbf{E}} T_{n w k}(\mathbf{f}, \mathbf{E})
$$

The maximum lifetime routing problem stated above can be formulated as a linear programming problem as shown below. Let $\hat{f}_{i, j}(\mathbf{f}, \mathbf{E})=f_{i, j} \cdot T_{n w k}(\mathbf{f}, \mathbf{E})$ be the aggregate information transferred over the link $(i, j)$ during the lifetime of the network. Since the data gathered by the sensor nodes is delivered to the collector nodes without any in-network compression or aggregation, we have

$$
\sum_{i \in M-C} r_{i} \cdot T_{n w k}(\mathbf{f}, \mathbf{E})=\sum_{i \in M-C, j \in C} \hat{f}_{i, j}(\mathbf{f}, \mathbf{E})
$$


Also since the data rates of the nodes are assumed to be fixed, the maximum lifetime problem can be stated as follows:

$$
\max _{\mathbf{f}, \mathbf{E}} T_{n w k}(\mathbf{f}, \mathbf{E}) \sim \max _{\mathbf{f}, \mathbf{E}} \sum_{i \in M-C, j \in C} \hat{f}_{i, j}(\mathbf{f}, \mathbf{E})
$$

subject to the following constraints:

$$
\begin{gathered}
\sum_{j \in M} \hat{f}_{j, i}+r_{i} \cdot T_{n w k}(\mathbf{f}, \mathbf{E})=\sum_{j \in M} \hat{f}_{i, j}, \forall i \in M-C, \\
\sum_{j \in M}\left\{\hat{f}_{i, j} \cdot \mathcal{E}_{t x}^{i, j}+\hat{f}_{j, i} \cdot \mathcal{E}_{r x}\right\}+r_{i} \cdot T_{n w k}(\mathbf{f}, \mathbf{E}) \cdot \mathcal{E}_{s} \leq E_{i}, \forall i \in M,
\end{gathered}
$$

$$
\begin{gathered}
\hat{f}_{i, j} \geq 0, \forall i, j \in M, \\
\hat{f}_{i, i}=0, \forall i \in M, \\
\hat{f}_{i, j}=0, \forall i \in C, j \in M
\end{gathered}
$$

In the above constraints and in subsequent analysis, it should be noted that $\hat{f}_{i, j}$ is indeed the function $\hat{f}_{i, j}(\mathbf{f}, \mathbf{E})$ though we avoid writing it explicitly for better readability. Equation (11) ensures that there is no flow from a sensor node to itself while (12) ensures that the collector nodes only gather sensor data and do not transmit any data back into the network.

When the initial energy of each node in the network is known, solving the above linear program results in the flow $f$ that maximizes the lifetime of the network. Most of the prior works consider nodes with equal initial energy. Instead if we consider the problem of optimal initial energy distribution among the nodes given a total energy budget $\left(E_{\text {total }}\right)$, then the above linear program can be extended by including the total initial energy constraint given by (2).

The linear program thus obtained simultaneously finds both the optimal initial energy allocation among the nodes and the optimal flow in the network that together maximize the lifetime of the network.

\section{Discussion AND Simulation RESUlts}

Some important observations made while solving the linear program stated above are in order. To proceed with solving the linear program, we first substitute the value of $r_{i} \cdot T_{n w k}(\mathbf{f})$ from (8) in the corresponding equation in (9) to obtain

$$
\sum_{j \in M}\left\{\hat{f}_{i, j} \cdot\left(\mathcal{E}_{t x}^{i, j}+\mathcal{E}_{s}\right)+\hat{f}_{j, i} \cdot\left(\mathcal{E}_{r x}-\mathcal{E}_{s}\right)\right\} \leq E_{i}, \forall i \in M
$$

We then substitute the value of $T_{n w k}(\mathbf{f})$ from (6) in each of the equations in (8) to obtain

$$
\sum_{j \in M} \hat{f}_{j, i}+\hat{r}_{i} \cdot \sum_{i \in M-C, j \in C} \hat{f}_{i, j}=\sum_{j \in M} \hat{f}_{i, j}, \forall i \in M-C
$$

where $\hat{r}_{i}$ is the normalized data rate of node $i$ such that $\sum_{i \in M-C} \hat{r}_{i}=1$. Thus it is the normalized data rates of the nodes and not their absolute rates that are required to solve the linear program, to obtain the optimal energy distribution among the nodes and the maximum throughput of the network. However, on computing the maximum throughput of the network the aggregate data rate of all the nodes is required to compute the lifetime of the network using (6).

As mentioned in Section I, the linear program formulation in [6] computes the maximum-lifetime flow for a given deployment of nodes over an information field with fixed source rates and fixed initial energies. In [10], it is shown through simulations that the lifetime of a grid-based average node deployment is close to the lifetimes of a significant percentile of random node deployments. This is because random node deployments can be viewed as perturbed versions of the gridbased deployment and with sufficiently large number of grids these perturbations result in only small deviations in the objective function. In our simulations too we use a grid-based node deployment and the conclusions drawn from them hold true for a large fraction of random node deployments.

We consider an information field of size $1000 \mathrm{~m} \times 1000 \mathrm{~m}$ with origin $(0,0)$ at the left bottom corner as shown in Fig. 1 and Fig. 2. The field is partitioned into a regular $8 \times 8$ grid, with each grid-unit assumed to have a single node placed at its center. The data rate of each node is equal to the volume under the information-rate density function within the grid-unit. In our simulations below, we consider two different informationrate density functions in the field: the first being a uniform density function as shown in Fig. 1 and the second a concave density function over the information field as shown in Fig. 2. The two functions are chosen such that the net volume or the total information rate in each case is equal to $1 \mathrm{Mbps}$.

In the simulations below, the total energy in the network $\left(E_{\text {total }}\right)$ is fixed at 1 Joule. The parameters in the energy model are taken from [9]: $\mathcal{E}_{r x}=135 \mathrm{~nJ} / \mathrm{bit}, \mathcal{E}_{s}=50 \mathrm{~nJ} / \mathrm{bit}$, and $\mathcal{E}_{t x}^{i, j}=\mathcal{E}_{t}+\mathcal{E}_{p a} \cdot d_{i, j}^{2}$, where $d_{i, j}$ is the distance between node $i$ and node $j, \mathcal{E}_{t}=45 \mathrm{~nJ} / \mathrm{bit}$ is the energy dissipated in the transmitter electronics. $\mathcal{E}_{p a}=10 \mathrm{pJ} / \mathrm{bit}-m^{2}$ is the transmit

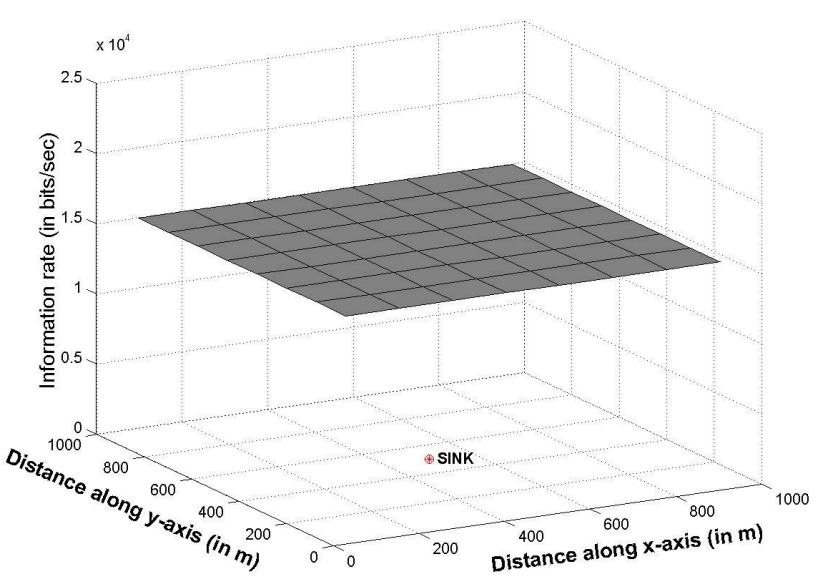

Fig. 1. Uniform information-rate distribution over the information field 


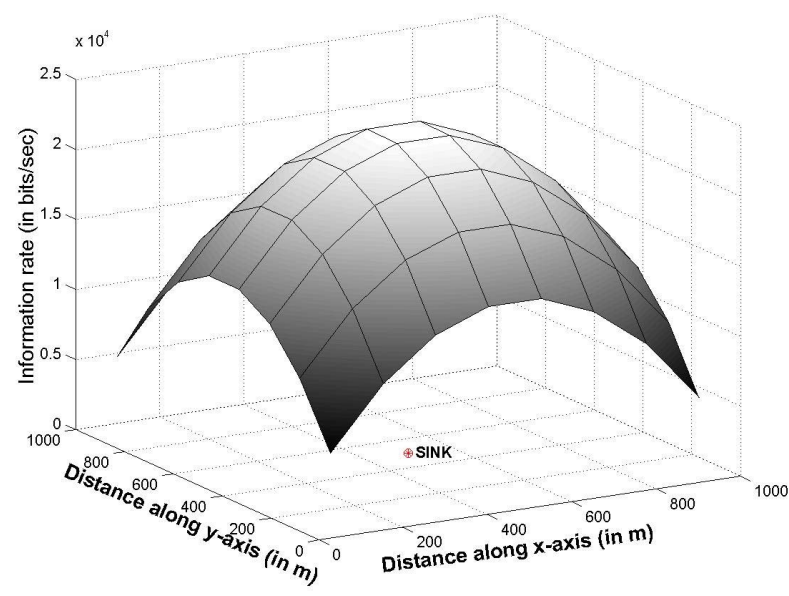

Fig. 2. Concave information-rate distribution over the information field

energy-per-bit required to achieve an SNR of $25 \mathrm{~dB}$ at the receiver when transmitting at $1 \mathrm{Mbps}$ over a $22 \mathrm{MHz}$ wide channel at $2.4 \mathrm{GHz}$, with path loss exponent $\alpha=2$, and assuming a receiver noise figure of $15 \mathrm{~dB}$.

\section{A. Uniform Node Distribution - Comparison of Network Life- times}

Consider a uniform distribution of nodes over the information field of Fig. 1 or Fig. 2. As mentioned earlier in this section, the grid-based model closely approximates the lifetime of a large fraction of networks based on uniform node distribution. Using this model, we compare the network lifetime obtained in two different cases, both with a total energy among all the nodes of 1 Joule. In the first case this energy is uniformly distributed among all the nodes, while in the second case the total energy is optimally distributed using the linear program formulation of the previous section. These two cases are repeated for the uniform and concave information-rate density functions shown in Fig. 1 and Fig. 2 respectively, resulting in a total of four different cases.

A single sink node in the network acts as a collector for the data generated at each of the sensor nodes. Fig. 3 plots the lifetime curves for the four different cases mentioned above by varying the position of the sink node in the network. The curves are plotted for eight different positions of the sink node in the network, the co-ordinates of the sink node being $(500,(n-5) \times 250)$ where $n$ is the index along the $\mathrm{x}$-axis in the figure.

As expected, Fig. 3 shows the relatively longer network lifetime achieved with the optimal energy distribution as against a uniform distribution. Depending on the position of the sink in the network, the improvement over the uniform energy distribution varies from $30 \%$ to nearly $100 \%$ with the optimal energy distribution. From Fig. 3, the variation in the network lifetime for different positions of the sink node also illustrates the importance of the location of the sink within the network. For the information-rate distributions in Fig. 1 and

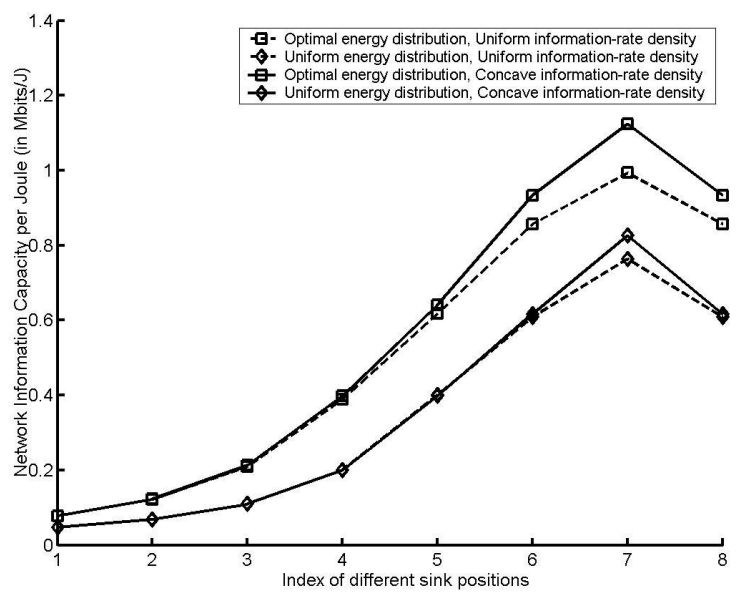

Fig. 3. Comparison of lifetimes with and without optimal energy distribution

Fig. 1 which are symmetric about the center of the information field, the lifetime of the network is maximized when the sink is located at the center of the information field. By placing the sink closer to regions with relatively higher data rates, the throughput-per-Joule of the network can be increased. For the more general case of an arbitrary information-rate density function, finding the optimal placement of one or more sink nodes within the field so as to maximize the network lifetime is an interesting issue for future study.

\section{B. Optimal node distribution}

We now address the important problem of finding the optimal node distribution in an information field given the information-rate density function and the position of the sink in the field. As mentioned in Section I, this problem has received attention in [10] where the authors search over a family of parametric distributions. Instead of searching over several different families of node distributions, we make use of the optimal energy formulation of Section III to obtain the optimal node distribution. The optimal energy distribution obtained from the linear program formulation can be considered to be equivalent to the allocation of energy to each node in a uniform node distribution. Yet another (possibly more practical) interpretation is that it is equivalent to the distribution of nodes with equal energy such that the number of nodes per unit area or equivalently the energy per unit area is equal to the optimal energy distribution. For the informationrate distributions shown in Fig. 1 and Fig. 2 and with the sink located at the center of the information field, we plot the optimal energy distributions or equivalently the optimal node distribution functions in Fig. 4 and Fig. 5. The optimal energy distribution for the case of the sink positioned at the corner $(1000,0)$ of the information field is shown in Fig. 6.

We now compare the performance of random networks generated using the optimal node distribution (shown in Fig. 7) with those generated using a uniform node deployment (shown in Fig. 8) when the sink is located at the center of 


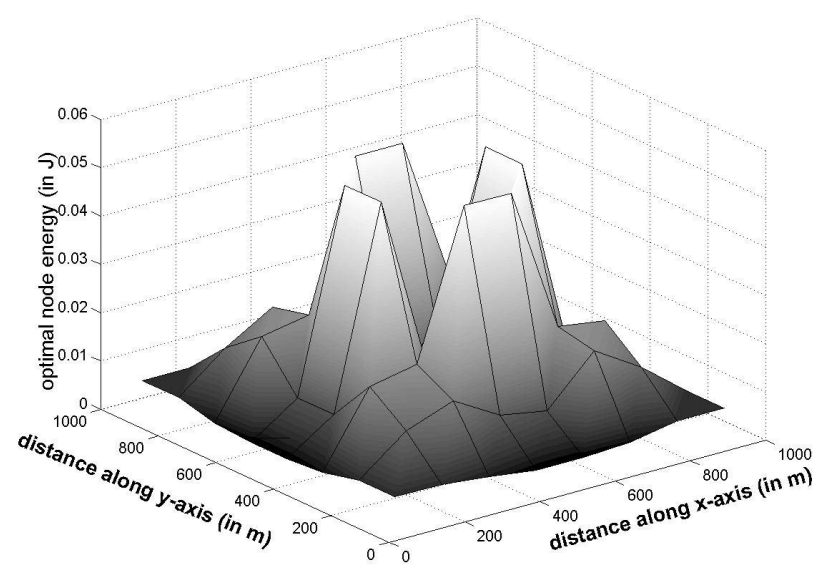

Fig. 4. Optimal energy distribution for uniform information-rate density and sink at the center of the field

the information field. Fig. 9 plots the information capacity per Joule of 100 random networks based on these two distributions deployed over an information field with concave informationrate density (Fig. 2). As can be seen from the figure, the average information capacity of networks with optimum node distribution is about $70 \%$ higher than the average information capacity of networks with uniform node distribution.

\section{CONCLUSion ANd Future Work}

In this paper, we considered a wireless sensor network deployed over an information field that is characterized by a grid-based information-rate density function. We presented a linear program based formulation for computing the optimal energy distribution and flow in the sensor network so as to maximize the lifetime of the network. Given the informationrate density in the underlying information field and the location of the sink, the optimal energy distribution gives the solution to the problem of finding the optimal initial node distribution that results in a network with maximum lifetime. The more general problem of placement of a set of sink nodes in the information field along with the sensor node distribution is a problem for future study.

\section{REFERENCES}

[1] S. Singh, M. Woo, and C. S. Raghavendra. Power-aware routing in mobile ad hoc networks. In Proc. ACM/IEEE Int. Conf. on Mobile computing and networking, pages 181-190, October 1998.

[2] V. Rodoplu and T. H. Meng. Minimum energy mobile wireless networks. In Proc. IEEE Int. Conf. on Communications, pages 1633-1639, June 1998.

[3] I. Stojmenovic and X. Lin. Power-aware localized routing in wireless networks. In Proc. IEEE Int. Parallel and Distributed Processing Symp., May 2000.

[4] R. Ramanathan and R. Rosales-Hain. Topology control of multihop wireless networks using transmit power adjustment. In Proc. IEEE INFOCOM, pages 404-413, March 2000.

[5] R. Wattenhofer, L. Li, P. Bahl, and Y. M. Wang. Distributed topology control for power efficient operation in multihop wireless ad hoc networks. In Proc. IEEE INFOCOM, April 2001.

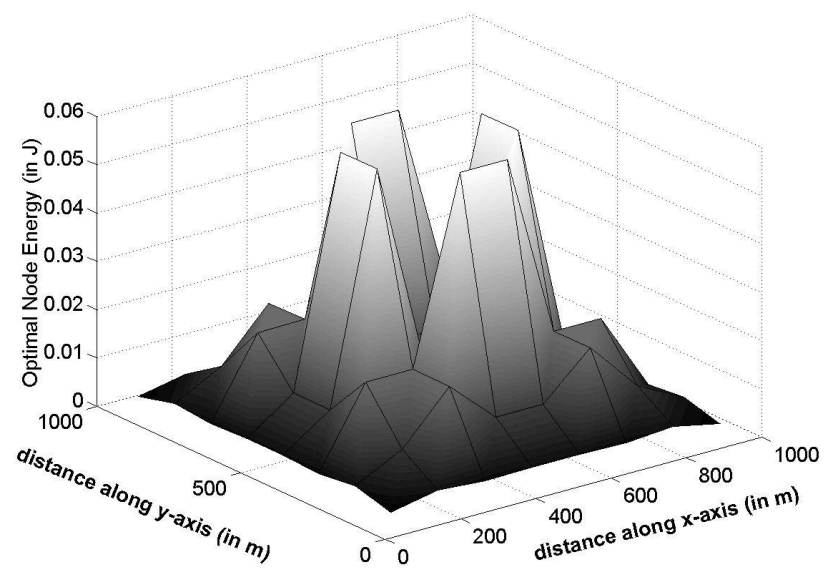

Fig. 5. Optimal energy distribution for concave information-rate density and sink at the center of the field

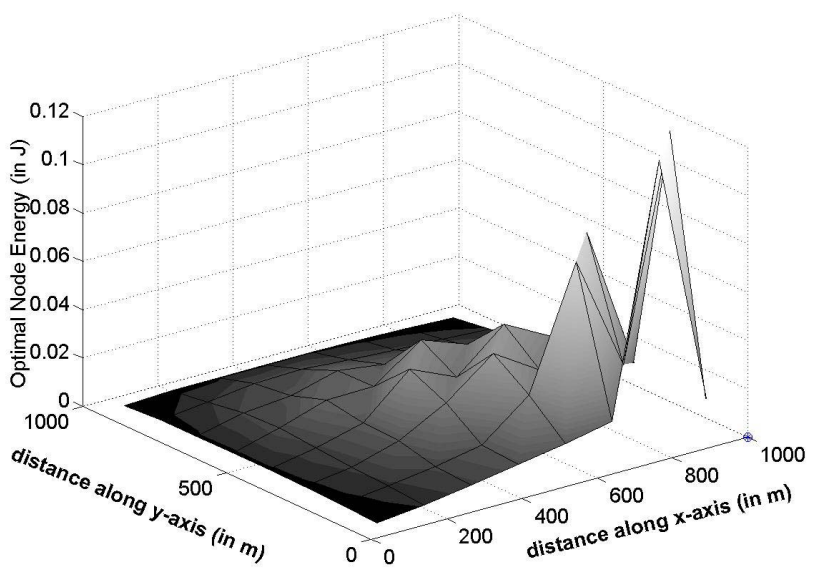

Fig. 6. Optimal energy distribution for concave information-rate density and sink at the corner of the field

[6] J. H. Chang and L. Tassiulas. Routing for maximum system lifetime in wireless ad-hoc networks. In 37th Annual Allerton Conference on Communications, Control and Computing, September 1999.

[7] J. H. Chang and L. Tassiulas. Energy conserving routing in wireless ad-hoc networks. In IEEE Infocom'00, March 2000. Tel Aviv, Israel.

[8] J. H. Chang and L. Tassiulas. Maximum Lifetime Routing in Wireless Sensor Networks. In Proceedings of Advanced Telecommunications and Information Distribution Research Program, 2000. College Park, MD.

[9] W. Heinzelman, A. Chandrakasan, and H. Balakrishnan. Energy-efficient routing protocols for wireless microsensor networks. In Proceedings of the 33rd Hawaii International Conference on Systems and Sciences, pages 1-10, 2000. Hawaii.

[10] E. J. Duarte-Melo, M. Liu, and A. Misra. Lifetime bounds, optimal node distributions and flow patterns for wireless sensor networks. In Technical Report, Electrical Engineering and Computer Science Department, University of Michigan, 2003.

[11] K. Dasgupta, M. Kukreja, and K. Kalpakis. Topology-aware placement and role assignment for energy-efficient information gathering in sensor networks. In Proc. 8th IEEE Symp. on Computers and Communication, pages 341-348, July 2003.

[12] F. Ordónez and B. Krishnamachari. Optimal Information Extraction in Energy-Limited Wireless Sensor Networks. IEEE JSAC, 22(6):11211129, August 2004. 


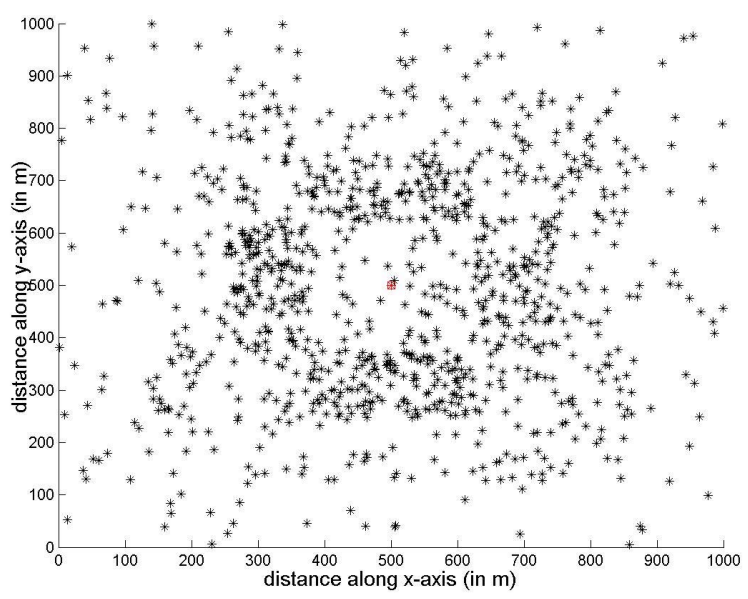

Fig. 7. Random node deployment based on the optimum node distribution

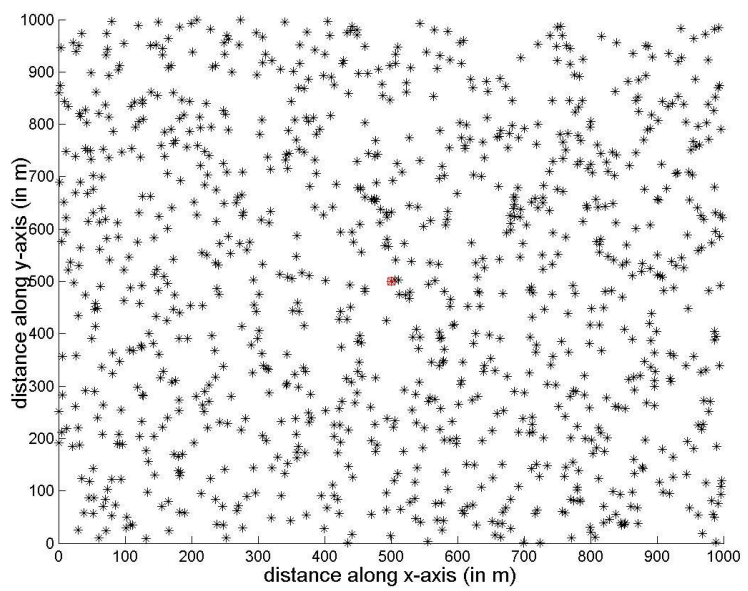

Fig. 8. Random node deployment based on a uniform node distribution

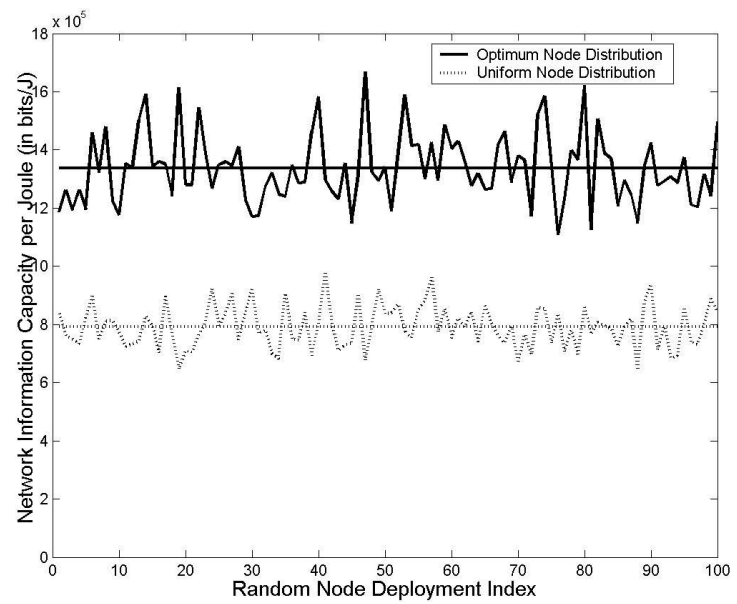

Fig. 9. Comparison of information capacity for random node deployments based on optimal and uniform node distributions 